\title{
A systematic review and meta-analysis: Female smokers,even former smokers lead higher incidence of psoriasis in the treatment of inflammatory bowel disease with biological agents.
}

\author{
Meiqi Yang \\ First Hospital of china medical university \\ Weixin Liu ( $\nabla$ drweixin@sohu.com ) \\ First Hospital of china medical university \\ Qiuping Deng \\ first hospital of China Medical University \\ Zeng Liang \\ first hospital of China Medical University \\ Qin Wang \\ first hospital of China Medical University
}

Research article

Keywords: inflammatory bowel disease(IBD), psoriasis, TNF-antagonist, infliximab, adalimumab, gender, smoke, incidence

Posted Date: May 11th, 2020

DOI: https://doi.org/10.21203/rs.3.rs-27277/v1

License: (c) (i) This work is licensed under a Creative Commons Attribution 4.0 International License. Read Full License 


\section{Abstract}

Background: At present, infliximab(IFX) and adalimumab(ADA) are the classic drugs for the treatment of moderate-severe inflammatory bowel disease(IBD), which prove effective for the disease control. However, the side effects need to be monitored during the therapy process, especially the paradoxical reaction of the skin system such as psoriasis, which could greatly benefit quality of patients' life.

Aim: In order to describe and analyze TNF-antagonist-induced psoriasis in patients with inflammatory bowel disease from the aspects of incidence, pathogenesis, distribution and type of lesions, prognosis and treatment, the incidence of psoriasis induced by Infliximab (IBD) and Adalimumab (ADA) in the treatment of Inflammatory Bowel Disease Disease (IBD) as well as the relationship between sex, smoking and the incidence of psoriasis were investigated.

Methods: Literatures in English language meeting the qualifications on Pubmeb, Embase, Web of Science, Google, and Geenmedical databases were searched . More than two co-authors evaluated the quality of the article and extracted the data respectively. The data obtained were statistically analyzed by statistical software of Revman and Stata.

Results: 1) The incidence of psoriasis was higher in ADA Group (OR 0.66, $\mathrm{P}<0.05,95 \% \mathrm{Cl}(0.52-0.84) ; 2)$ The incidence of psoriasis was higher in females than in males (OR 1.54, $\mathrm{P}<0.05,95 \% \mathrm{Cl}(1.27-1.86)$ ) ; 3) Smoking increased the incidence of psoriasis (OR 1.79, $\mathrm{P}<0.01,95 \% \mathrm{Cl}(1.42-2.24)$; 4$) \mathrm{Psoriasis}$ is mainly distributed in the Scalp, Palmoplantar, skin folds and limbs, and often occurs in the genital organs, the pathogenesis of which is not completely clear; the interval of medication is more than one year, and the interval of medication using IFX is longer than that of ADA Group; most cases can be relieved by local hormone, phototherapy or systemic hormone therapy without changing the strategy of biological agents.

Conclusion: The incidence of psoriasis induced by TNF-antagonists is higher in many autoimmune diseases. The frequency of reported in inflammatory bowel disease is higher than other autoimmune diseases, and the safety of ADA Treatment for IBD is higher than that of IFX The incidence of psoriasis is higher in women than in men; the incidence of psoriasis is significantly higher in smokers / ex-smokers than in non-smokers. The interval of drug use of TNFantagonists may be a predictive factor for the prevention and treatment of psoriasis, and there are differences among different kinds of TNF-antagonists. Change of therapeutic strategy of TNF-antagonists is not recommended in the event of psoriasis.

\section{Background}

IBD is a chronic, nonspecific inflammatory disease caused by autoimmune disorders of the colorectal mucosa, with recurrent inflammatory lesions[1, 2].Currently, IBD include Ulcerative Colitis (UC) , Crohn (CD) , and undifferentiated types, which severely affect the quality of life of patients and often require ongoing combination therapy. Tumor necrosis factor (TNF) antagonists play an important role in the treatment of various autoimmune inflammatory diseases such as rheumatoid arthritis, inflammatory bowel disease, psoriasis, etc. The 2018 inflammatory bowel disease consensus suggests that biological agents should be considered for the treatment of moderate to severe ulcerative colitis. According to the American Gastroenterological Association (AGA) clinical guidelines, patients with moderate to severe disease who do not respond to mesalazine therapy and who do not respond well to hormonal or immunosuppressive agents should consider the use of biological agents [3]. Tumor necrosis factor (TNF) is expressed in a large number of intestinal mucosal cells in IBD patients, which is directly involved in the occurrence and progression of the Disease[1] $\mathrm{TNF}$-antagonists are effective for refractory UC and CD patients with fistula and sinus formation[2]. TNF-antagonists are not only used in the treatment of inflammatory bowel disease, but also commonly used in a variety of autoimmune diseases[4]. The efficacy of TNF-antagonists has been confirmed[5]. With the increasing use of TNF-antagonists, the occurrence of drug-induced side effects can be overlooked. Psoriasis is an autoimmune disease that seriously affects quality of people's life, which usually appears after treatment with TNF-antagonists[6-9]. There are also cases of pathogenic infection, vasculitis, drug induced lupus, eczema, erythema multiform and various skin malignancies[10]囚of all retrospective, cohort and case-control study studies, psoriasis (PSO) was most frequently reported. TNF-antagonists are widely used in the treatment of psoriasis, but with the increase of contradictory reactions, the safety of TNF-antagonists is worth testing. Psoriasis is an autoimmune skin disease in which abnormal t-cell-mediated Keratinocytes overproliferate and differentiate abnormally [11-13]. Studies have shown that TNF-antagonistinduced psoriasis and primary psoriasis are not identical in histopathology and immunohistology[14]. There was a proportion meta-analysis for the correlation between psoraisis and IBD prove that significant bidirectional associations between them[15].

At present, the most widely used TNF-antagonists are Infliximab (IFX) and Adalimumab (ADA). IFX was first used in the treatment of adult and juvenile IBD, and ADA is mainly utilized in adult IBD. The use of TNF-antagonists in children and adolescents with IBD is increasing[16], and the effectiveness and safety of TNF-antagonists are summarized, discussed and analyzed in various literatures. The increased frequnency of psoriasis in IBD patients with biological agents had been reported[15]. It's controversial for the prevalence of TNF antagonists induced psoraisis between different biological agents and different genders and smokers or not. We aimed to describe the prevalence and association between psoraisis and usage of biological agents and relevant risk factors through a systematic review and meta-analysis.

\section{Methods}

\section{Search strategy}

This study was conducted in accordance with the Preferred Reporting Items for Systematic Reviews and Meta-Analyses (PRISMA). By collecting 2000-to-date literatures in Pubmed, Embase, Web of Science, CHN and other databases, we set up the key words for searching: Inflammatory Bowel disease, psoraitic,PSO, IFX, ADA, paradoxical reaction, adverse reaction, adult, pediatric, etc. , searching in title / abstract, by searching keywords such as "and" , "or" and by using Mesh keywords and advanced search, we also checked some references with high quality and screened out the literatures that meet the standard. After the preliminary screening, more than 500 literatures were initially selected. The literature was further screened according to the inclusion and exclusion criteria. 


\section{Inclusion and exclusion criteria}

Literatures that met the following inclusion criteria were included in the Meta analysis: 1) It was observational; 2) the data were complete; the incidence of psoriasis was clear; and for Case-control study, there was complete information on the experimental and control groups.

Exclusion criteria of the literature: 1) subjects with other autoimmune diseases or with more severe systemic diseases; 2) the experiment was poorly designed or lacked a control group; 3 ) psoriasis was not pathologically confirmed; 4) The score of the article was less than 6 after the evaluation of the quality of NOS;

5) the same article was published repeatedly.

By summarizing the conclusions of each study, the incidence, the distribution of psoriasis, the time interval between onset and medication, and the prognosis of psoriasis was analyzed. We extracted the required data: (1) General Data: first Author's name, year of publication, population nationality, inclusion sample size, gender distribution, distribution of IFX and ADA use, smoking history; (2) Outcome data: sample size, adjuvant therapy, lesion coverage, and management of psoriasis in the psoriasis group and the control group (Table 1). The NOS evaluation scale was used to evaluate the quality of the collected data. The results were shown in Fig. 1

\section{Statistical analysis}

The OR value was calculated by the fixed effect model $\triangle \mathrm{FEM} \nabla$ method, and the incidence ratio between the experimental group and the control group was estimated. The heterogeneity between the studies was calculated by Cochrane's Q-test and $\mathrm{I}^{2}$ static[17], of which $\mathrm{I}^{2}$ describes the total variation across studies due to heterogeneity rather than chance. Formula for $\mathrm{I}^{2}$ :

$I^{2}=100 \% * \otimes Q-d f \otimes / Q$

$\mathrm{Q}$ is the Cochran's heterogeneity data, and df is the degree of freedom. The funnel plot was used to analyze the publication bias(shown in Fig 11). However, due to the small number of articles and strict quality evaluation, combined with the funnel plots, it can be considered that there is no publication bias. Revman and Stata, version 12.0 , were used for all data analysis. $\mathrm{P}<0.05$ was considered statistically significant.

\section{Result}

\section{Study characteristics}

Detailed description of screening steps (as shown in Figure 1): more than 500 studies were obtained through the preliminary screening of topics and abstracts. Of these results, 48 studies met the inclusion criteria, except for 5 papers that could not obtain full text. After reading the full texts, 27 papers were excluded according to the exclusion criteria. After the quality evaluation again, we finally included 12 studies in this meta-analysis. Table 1 shows the characteristics of the 12 literatures. Table 2 shows the summary of results.

\section{Summary results}

1. Comparison of the Incidence of Psoriasis induced by IFX and ADA

The incidence of psoriasis induced by IFX and ADA was analyzed by meta-analysis. The patients treated with IFX and ADA were regarded as the experimental group and control group, respectively, the incidence of psoriasis was compared between the two drugs. After statistical analysis of the data, the results showed: $\mathrm{OR}=0.66,95 \% \mathrm{Cl}(0.52-0.84), \mathrm{P}<0.05$, there was statistical significance, indicating that the incidence rate of the IFX group was 0.66 times than the ADA group, heterogeneity: $Q=11.35 P=0.12,2^{2=} 38 \%$, shown in figure 3. Further sensitivity analysis to identify heterogeneous sources found that $S H I L P A$ SRIDHAR ET AL's study was different from other studies in that it was a group of pediatric patients, which may contributed to the heterogeneity of the results. The results were as follows: $\mathrm{OR}=0.60,95 \% \mathrm{Cl}(0.46-0.77)$, heterogeneity results: $\mathrm{Q}=6.05 \mathrm{P}<0.42, \mathrm{I}^{2}=21.0 \%$, shown in fig4. Therefore, this study can be considered as the source of heterogeneity[18]. No significant difference in the incidence and heterogeneity of psoriasis was found in the sensitivity analysis of other studies.

2. Time Interval of Drug Administration for Psoriasis induced by TNF-antagonist

It is very important for the occurrence and prognosis of psoriasis to master the interval of Drug Administration of psoriasis and TNF-antagonist, which can closely monitor the skin changes of patients in the high incidence period of psoriasis. In most of the studies, TNF-antagonist-induced psoriasis occurred more than 1 year after administration[10, 11, 13, 19-25], and in a few of the studies, the interval of administration was less than 1 year[26]. Among them, 3 studies reported the interval of administration in IFX and ADA groups respectively[13, 19, 21]. The IFX group had a longer interval than the ADA group in the two of three studies[19, 21]; the ADA group in one study had a slightly longer interval than the IFX group, and the interval was less than one year (10 months) for the IFX group[13]. No study has mentioned the relationship between the interval of administration of TNF-antagonists and the incidence of psoriasis.

3. Effect of Sex on the Incidence of Psoriasis induced by TNF-antagonists $\rrbracket$

The incidence of psoriasis in different sex groups of IBD including 9 literatures, respectively. The incidence of different sex groups after treatment with TNFantagonist, and the results were statistically significant: $\mathrm{OR}=1.54,95 \% \mathrm{Cl}(1.27-1.86), \mathrm{P}<0.01$. The heterogeneity results: $\mathrm{Q}=14.53 \mathrm{P}=0.07, \mathrm{I}^{2}=45 \%$, fig5. Further sensitivity analysis indicated that $\mathrm{OR}$ value of Jean Ois Rahier et al study was lower $(\mathrm{OR}=0.32,95 \% \mathrm{Cl}(0.08-1.19))$, which may affect the stability and reliability of the results and increase the heterogeneity of the results. After excluding the study, the results were analyzed again. The results showed that the heterogeneity decreased significantly $\left(Q=8.90, P=0.26, I^{2}=21 \%\right)$, shown in fig6. Most of the subjects in this study were patients with Crohn's disease, and the 
control group were patients with other skin diseases besides psoriasis. Some patients may have multiple skin lesions, therefore there may be cross and interaction between different types of skin lesions.

The study was divided into two subgroups: Adult group and Teenager group. The results were as follows: $\mathrm{OR}_{\mathrm{Adult}}$ group $=1.52,95 \% \mathrm{Cl}(1.24-1.87), \mathrm{P}<0.05$, heterogeneity: $\mathrm{I}^{2=} 65 \%, \mathrm{OR}$ Teenager group $=1.74,95 \% \mathrm{Cl}(1.07-2.82), \mathrm{P}<0.05$, heterogeneity: $\mathrm{Q}=0.92, \mathrm{P}=0.63, \mathrm{I}^{2=} 0 \%$, shown in fig7. In the two subgroups, the conclusion is the same: Psoriasis is higher in the female population, and the $\mathrm{OR}_{\text {Teenager group }}>\mathrm{OR}$ Adult group. The conclusion is that the incidence of psoriasis is higher in the pediatric IBD patients treated with TNF-antagonist.

\section{The effects of smoking on psoriasis}

The results showed that the incidence of psoriasis in smoking / ex-smoking Group was significantly higher than that in non-smoking group: OR=1.79, $\left.\mathrm{P}<0.01,95 \% \mathrm{Cl}(1.42-2.24), \mathrm{I}^{2=}=8 \%\right)$, shown in fig8. Psoriasis is more common in smokers / ex-smokers of IBD treated with TNF-antagonists. Smoking has been reported to be a risk factor for TNF-antagonists-induced psoriasis [27] , which increased the incidence of psoriasis, but some researchers suggested that there was no significant difference between smokers and non-smokers in the incidence of psoriasis induced by TNF-antagonists [28]. From this analysis we may draw a conclusion: smoking is a major risk factor for IBD, especially for ulcerative colitis patients. Quitting smoking is one of the interventions for IBD patients to control disease progression and prevent disease recurrence.

\section{Distribution of Lesions}

Statistically, skin lesions usually do not appear as a single form, and multiple skin lesions can occur simultaneously [31]. More than 80\% of the reported TNFantagonist-induced psoriasis lesions involved more than 2 sites of body[13,24, 25]. According to the literature, the skin lesions of psoriasis induced by TNFantagonists were mainly distributed in scalp, metacarpal, limbs and skin folds $[13,19,29,30]$. The most common lesions were located in the scalp and palmoplantar regions[31] with equal incidence and the skin fold was the second favourable region[9]. Studies by Pugliese et Al. found that the sacroiliac region, the extensor sides of the knee and elbow were also the most frequently affected areas [32]. Its clinical manifestation has many types, of which the general manifestation includes the plaque type, granular type and pustule type [33]. The plaque type was the most common manifestation [34, 35].

\section{Treatment and Prognosis of Psoriasis}

At present, it is believed that the treatment of psoriasis induced by TNF-antagonist should be evaluated from the severity of psoriasis, the extent and degree of lesions, the impact on patients' quality of life and the impact on patients' psychology, etc. Whether to use TNF-antagonist is a key problem in the treatment of psoriasis. It is extremely important to monitor the condition of patients and the changes of skin lesions [36]. It is not necessary to stop using TNF-antagonists in patients with mild psoriasis who have not yet had a significant impact on normal quality of life [37, 38]. Local application of steroids is often the preferred treatment for most lesions [18, 42]. Of the 12 studies, totally 692 patients suffer psoriasis induced by TNF-antagonists, and 137 patients stopped or switched to biological agents, and more than $50 \%$ of them had good prognosis; Freling e et Al. concluded from a 14 year single center large sample study that about $50 \%$ of patients were able to control their condition without stopping their medication through topical medicine, and about $20 \%$ needed to stop their medication [21]. According to statistics, about $40 \%$ of patients can relieve skin lesions through local hormones, while $20 \%$ of patients can relieve the condition through ultraviolet light therapy. Wolf D et Al. analyzed the clinical outcomes of patients in group 377 (754 cases) who withdraw or switch of TNF-antagonist and those who continued TNF-antagonist. The clinical recurrence rate was higher in 377 groups(754 cases) who stopped or converted TNF-antagonist for nonmedical reasons, and the frequency of in-hospital treatment, emergency treatment and out-patient treatment was higher than that of continued use of TNFantagonist [39].

\section{Pathogenesis of psoriasis}

The occurrence of psoriasis is closely linked to autoimmune factors and may also be related to genetic factors. So all studies exclude those with personal and family history to ensure the reliability of the results. Some studies suggest that psoriasis may be a prevalent side effect of TNF-antagonists, also known as class effects [11]. The pathogenesis of psoriasis induced by TNF-antagonists is still not completely clear. According to current reports, there are several mechanisms: 1) under normal conditions, the balance of TNF-a and IFN- $\gamma$ is maintained in the body, which plays a role in regulating the body's inflammatory response. When TNF- $\alpha$ is greatly inhibited, the balance between TNF- $\alpha$ and IFN- $\gamma$ is destroyed, resulting in an increase in the production of interferons (IFN- $\gamma$ ) by dermal plasmacytoma dendritic cell, and excessivly promotes the migration of CD $8+\mathrm{t}$ cells to the epidermis, leading to activation and maturation of dendritic cell cells, thus inducing the occurrence of the autoimmune response[30, 40]; 2) the Interleukin (II)-23 / T-helper (TH)-17 axis also plays an important role in the pathogenesis of TNF antagonist-induced psoriasis. IFN induced TH17 cell proliferation and IL-23 induced the release of large amounts of interleukin-17(IL-17) from helper TH17 and TH1 cells, resulting in the disruption of cytokine balance in vivo [10], Which can lead to psoriasis [24, 29, 41], It may be related to IFN induced gene overexpression[22]. There are also a small number of people who believe that IBD patients with Cytokine receptor mutation, which leads to psoriasis [18]; 3) treatment with TNF-antagonists increases the risk of infection, and exposure to infected organisms increases the risk of psoriasis, especially pustular psoriasis [37]; 4) many autoimmune diseases originate at the gene level, TNF-antagonist-induced psoriasis may be associated with the pathogenesis of psoriasis, and patients using TNF-antagonists are more genetic predisposition [42]. It may also be related to the genetic variation of IFN, and there is evidence to support the role of the $x$ chromosome in autoimmune diseases[43].

\section{Evaluation of Heterogeneity}

We analyze the heterogeneity through sensitivity analysis and subgroup analysis. SHiPA et al mainly analyze the drug-induced psoriasis in teenagers which lead to the heterogeneity. After exclude the study the heterogeneity turn out to be $Q=6.05, P=0.42, I^{2}=1.0 \%$. It's obvious that this study is the source of heterogeneity. In the study of the relationship between gender and the incidence of TNF-antagonist induced psoriasis, we conduct subgroups according to age, 
and divided the 9 literatures into two groups, the Adult group: $\mathrm{OR}=1.6095 \% \mathrm{Cl}(1.291-1.983), \mathrm{n}=6, \mathrm{P}<0.05$, statistically significant, heterogeneity: $\mathrm{Q}=18.40, \mathrm{P}$ $=0.002, I^{2}=72.8 \%$, the Pediatric group: $\mathrm{OR}=1.736,95 \% \mathrm{Cl}(1.070-2.816), \mathrm{n}=3, \mathrm{P}<0.05$, statistically significant, heterogeneity: $\mathrm{Q}=0.92, \mathrm{P}=0.063, \mathrm{I}^{2}=0.0 \%$. In the two groups, the incidence is higher in female, especially in pediatric group. It can be seen that the heterogeneity mainly comes from the adult group(Figure 7).

In the study of smoking and drug-induced morbidity, we conducted subgroup analysis according to the sample size, and found that the heterogeneity mainly come from the Large sample size group: $Q=5.25, P=0.073, I^{2}=61.9 \%$. Small sample size group: $Q=0.09, P=0.958, I^{2}=0.0 \%$, shown in fig9. Considering that the heterogeneity may come from the study of Ivan Guerra and MD et al, we removed both two studies, and the results were $\mathrm{OR}=1.4,95 \% \mathrm{Cl}(1.085-1.806)$, heterogeneity: $Q=4.00, P=0.406, I^{2}=0.0 \%$.

\section{Publication bias}

By conducting the funnel plots, basically symmetrical distribution of the studies can be seen are. We can ignore the publication bias. Accompanied by the Galbriarth chart, shown in fig10, it can be considered that the publication bias is not large enough to affect the research results.

\section{Discussion}

The meta-analysis showed significant association between drug induced psoriasis and IBD. By comparing the drug safety of IFX and ADA, we found that the prevalence of new onset psoriasis in ADA is lower than IFX. The incidence of psoriasis in ADA is 1.6 times that of IFX. This was in accordance with previous cases of psoriasis induced by ADA more than IFX[44]. The usage duration maybe one of inpact factors of paradoxical reaction. The median timespan between initiation of therapy to occurance of psoriasis was two to six monts[45]. The usage duration of IFX is longer than the ADA for the treatment of CD than UC, especially in developing countries[12]. The efficacy of IFX and ADA was similar in a pediatric CD cohort study and associated with the age of diagnosis. The number of AEs was tolerable and more relative to IFX[46]. There was a difference between UC and CD. Genetic overlap between psoraisis and IBD may be responsible for the distinction between IFX and ADA[47]. The ability of selective function to inflammatory cytokines of biological agents and disruption of the cytokine milieu might be relavent to the occurrence of autoimmune diseases. Anita Afzali et al. analyzed the relationship and the influencing factors between TNF inhibitors and psoriasis in IBD patients and proved that there is no significant difference IFX and ADA[20, 25, 28]. D.Pugliese et al. concluded to the same conclusion through an analysis of previously published data[32]; However, as long as the ADA was widly used in IBD patients, more attention on its safty and new studies showed the prevalence of AEs is higher in ADA than IFX[18, 26].

The meta-analysis concluded that women were stronger predictors of sex $(\mathrm{OR}=1.54)$ and had a 1.54 times higher incidence of psoriasis than male. Women with IBD are more likely to develop psoriasis induced by TNF antagonist in amount of studies[20,29, 32]. Hence it is necessary to pay more attention to the psoriasis inpact on the quality of life and mental health in female patients. Contrarily, some studies have suggested that sex is not a risk factor for psoriasis induced by TNF antagonists even concluded that the incidence of psoraisis is higher in men[1]. The difference between conclusions may be related to the ethnity and nation of the study subjects. The psoriasis is susceptible to the women for relating with the psychological factors. The incidence of psoriasis was higher in female in all studies for pediatric patients, while only one study reported lower incidence in female[28]. There are significant differences in the incidence of psoriasis in the different genders in the pediatric group (7-17 years) treated with IFX [46]. And in most cohort studies of pediatric patients, TNF antagonist induced psoriasis is more predisposed to the female population[18, 48]. In our study, $\mathrm{OR}_{\text {Teenager group }}$ is higher than $\mathrm{OR}_{\mathrm{Adult}}$ group in the analysis of the association between different gender groups.

The incidence of psoriasis in IBD patients treated with TNF-antagonist was higher than that of non-smokers[10, 13]. Smoking has been identified as a major risk factor for TNF-antagonist-induced psoriasis in IBD patients[32], smokers / ex-smokers are at high risk for drug-induced psoriasis. Lauren A et al. discovered obesity is another risk factor for psoriasis in addition to smoking through designed case-control study[24].

The mechanism leading to the induction or exacerbation of psoriasis by TNF antagonists is unclear[7]. The change of therapy regimen is important for prognosis when the TNF antagonists induced psoriasis appearred. Justin $\mathrm{M} \mathrm{K}$ et al. concluded the best choice for TNF antagonists induced psoriasis is discontinuation of biologic agents[34]. The majority of IBD patients suffered from relapse or aggravation after withdraw or switch of biological agents[33]. However, some scholars believed that the continuation of biological agents will not affect the recovery of drug-induced psoriasis, and the lesions can recover under the local hormone or topical dermatologic medicine. There was no adequate evidence to prove that discontinuation of TNF antagonists for noninfectious skin lesions can alleviate condition[49]. D Pugliese et al. first reported that the combination of biological agents with immunosuppressants could reduce the risk of psoriasis[32, 50].

Studies have shown that drug concentration and efficacy is proportional[51]. Hence it is necessary to monitor the relationship between concentration and side effects. High concentration of biological agents may be one of the causes for AEs[21]. Therefore, some people try to find the Minimum Effective Dose (MED) by monitoring AEs in the treatment, so as to avoid the side effect to the greatest extent[52]. From a clinical point of view, identification is most important for the drug-induced AEs as soon as possible[9]. Many clinical manifestations can be used to predict side effects may be inadvertently ignored by physicians. The genetic expressiom markers were studied for prediction of TNF antagonists induced psoriasis[53]. Some studies suggested that suspending the use of biological agents or extending the duration of drug therapy has no effect on the relief of drug-induced psoriasis[54-56]. It is worth noting that psoriasis can also occur in IBD extraintestinal complications should be differentiated with TNF antagonists induced psoriasis[57]. Drug-induced complications are more common than extraintestinal complications. In clinical practice, we must strengthen health education for patients with relapsed condition and improve patients' compliance. The long-time follow up is necessary for IBD patients to ask for the effects and AEs to make an accurate judgment and guide the further regimens. Long-term management is advised to minimize the potential adverse effects of TNF antagonist therapy. 


\section{Conclusion}

The incidence of psoriasis induced by TNF-aantagonists is higher than other autoimmune diseases, and the safety of ADA Treatment for IBD is better than that of IFX. The incidence of psoriasis is higher in female than in male; the incidence of psoriasis is significantly higher in smokers / ex-smokers than in nonsmokers. The interval of drug use of TNF-antagonists may be a predictive factor for the prevention and treatment of psoriasis. There are differences among different types of TNFaantagonists and it seems no need of changing the therapeutic strategy of TNF antagonists in the event of psoriasis.

\section{Limitation}

All the literatures included in this meta-analysis are retrospective literatures. Although the three co-authors evaluated the quality of the article separately through the NOS evaluation list, the subjectivity of authors affect the quality evaluation results. The data sources are all from the electronic medical records of different hospitals or database could be incomplete. This paper tried to select the articles with complete data and screen out the low-quality literatures. In addition, all the studies included are from European and American countries, and basically do not contain the studies of Asian and African countries.

\section{Abbreviations}

IFX: infliximab

ADA: adalumumab

IBD: inflammatory bowel disease

UC: ulcerative colitis

CD: crohn disease

PSO: psoriasis

AGA: American Gastroenterological Association

IL: interleukin

FEM: fixed effect model

TNF: tumor necrosis factor

IFN: interferon

MED: Minimum Effective Dose

CAEs: Cutaneous Adverse Events

AEs: Adverse Events

\section{Declarations}

Ethics approval and consent to participate: This type of article is not required for ethics approval and consent to participants.

Consent for publication: Not Applicable

Availability of data and material: Not applicable. All the data we used can be searched on the Web.

Competing interests: There is no any financial and non- financial competing interests.

Funding : No funding.

Authors' contributions

"All authors have contributed to and approve the finel version of the article. YMQand LWX participated in the study design and wrote the main manuscript text, and DQP, LZ and WQ participated in the data analysis. All authors reviewed the manuscript".

Acknowledgements: Not Applicable

Authors' information: Meiqi Yang, from the first hospital of China Medical University, e-mail: meiqiyang@sohu.com

Weixin Liu, from the first hospital of China Medical University, e-mail: drweixin@sohu.com

Qiuping Deng, from the first hospital of China Medical University, e-mail: 13593702284@139.com 
Zeng Liang, the first hospital of China Medical University, e-mail: 3457114735@qq.com

Qin Wang, from the first hospital of China Medical University, e-mail: 13206237848@sohu.com

\section{Reference}

[1] Bae JM, Lee HH, Lee BI, Lee KM, Eun SH, Cho ML, et al. Incidence of psoriasiform diseases secondary to tumour necrosis factor antagonists in patients with inflammatory bowel disease: a nationwide population-based cohort study[J]. Alimentary pharmacology \& therapeutics. 2018,48(2):196-205.

[2] Kip KE, Swoger JM, Grandinetti LM, Barrie AM, 3rd, Greer JB, Regueiro MD. Tumor necrosis factor alpha antagonist-associated psoriasis in inflammatory diseases: an analysis of the FDA adverse event reporting system[J]. Inflammatory bowel diseases. 2013,19(6):1164-72.

[3] Ko CW, Singh S, Feuerstein JD, Falck-Ytter C, Falck-Ytter Y, Cross RK, et al. AGA Clinical Practice Guidelines on the Management of Mild-to-Moderate Ulcerative Colitis[J]. Gastroenterology. 2019,156(3):748-64.

[4] Perez-De-Lis M, Retamozo S, Flores-Chavez A, Kostov B, Perez-Alvarez R, Brito-Zeron P, et al. Autoimmune diseases induced by biological agents. A review of 12,731 cases (BIOGEAS Registry)[J]. Expert opinion on drug safety. 2017,16(11):1255-71.

[5] Tracey D, Klareskog L, Sasso EH, Salfeld JG, Tak PP. Tumor necrosis factor antagonist mechanisms of action: a comprehensive review[J]. Pharmacology \& therapeutics. 2008,117(2):244-79.

[6] Verea MM, Del Pozo J, Yebra-Pimentel MT, Porta A, Fonseca E. Psoriasiform eruption induced by infliximab[J]. The Annals of pharmacotherapy. 2004,38(1):54-7.

[7] Collamer AN, Battafarano DF. Psoriatic skin lesions induced by tumor necrosis factor antagonist therapy: clinical features and possible immunopathogenesis[J]. Seminars in arthritis and rheumatism. 2010,40(3):233-40.

[8] Cullen G, Kroshinsky D, Cheifetz AS, Korzenik JR. Psoriasis associated with anti-tumour necrosis factor therapy in inflammatory bowel disease: a new series and a review of 120 cases from the literature[J]. Alimentary pharmacology \& therapeutics. 2011,34(11-12):1318-27.

[9] Puig L. Paradoxical Reactions: Anti-Tumor Necrosis Factor Alpha Agents, Ustekinumab, Secukinumab, Ixekizumab, and Others[J]. Current problems in dermatology. 2018,53:49-63.

[10] Andrade P, Lopes S, Gaspar R, Nunes A, Magina S, Macedo G. Anti-Tumor Necrosis Factor-alpha-Induced Dermatological Complications in a Large Cohort of Inflammatory Bowel Disease Patients[J]. Digestive diseases and sciences. 2018,63(3):746-54.

[11] Guerra I, Algaba A, Perez-Calle JL, Chaparro M, Marin-Jimenez I, Garcia-Castellanos R, et al. Induction of psoriasis with anti-TNF agents in patients with inflammatory bowel disease: a report of 21 cases[J]. Journal of Crohn's \& colitis. 2012,6(5):518-23.

[12] Fiorino G, Allez M, Malesci A, Danese S. Review article: anti TNF-alpha induced psoriasis in patients with inflammatory bowel disease[J]. Alimentary pharmacology \& therapeutics. 2009,29(9):921-7.

[13] Guerra I, Perez-Jeldres T, Iborra M, Algaba A, Monfort D, Calvet X, et al. Incidence, Clinical Characteristics, and Management of Psoriasis Induced by Anti-TNF Therapy in Patients with Inflammatory Bowel Disease: A Nationwide Cohort Study[J]. Inflammatory bowel diseases. 2016,22(4):894-901.

[14] Stoffel EM, H ; Riedl, E ;. <Pub.Med._Emmanuella_Guenova_2017-Analysis_of_anti-TNF-induced_skin_lesions.pdf>[J]. 2018.

[15] MD FAMHGT. Global prevalence and bidirectional association between psoriasis and inflammatory bowel disease - A systematic review and meta-

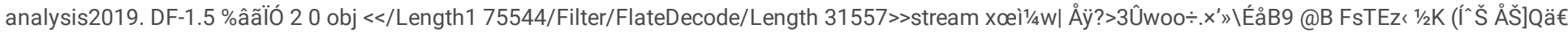
p.

[16] Patel AS, Suarez LD, Rosh JR. Adalimumab in pediatric Crohn's disease[J]. Immunotherapy. 2016,8(2):127-33.

[17] Chen B, Benedetti A. Quantifying heterogeneity in individual participant data meta-analysis with binary outcomes[J]. Systematic reviews. 2017,6(1):243.

[18] Sridhar S, Maltz RM, Boyle B, Kim SC. Dermatological Manifestations in Pediatric Patients with Inflammatory Bowel Diseases on Anti-TNF Therapy[J]. Inflammatory bowel diseases. 2018.

[19] Rahier JF, Buche S, Peyrin-Biroulet L, Bouhnik Y, Duclos B, Louis E, et al. Severe skin lesions cause patients with inflammatory bowel disease to discontinue anti-tumor necrosis factor therapy[J]. Clinical gastroenterology and hepatology : the official clinical practice journal of the American Gastroenterological Association. 2010,8(12):1048-55.

[20] Cleynen I, Van Moerkercke W, Billiet T, Vandecandelaere P, Vande Casteele N, Breynaert C, et al. Characteristics of Skin Lesions Associated With AntiTumor Necrosis Factor Therapy in Patients With Inflammatory Bowel Disease: A Cohort Study[J]. Annals of internal medicine. 2016,164(1):10-22. 
[21] Freling E, Baumann C, Cuny JF, Bigard MA, Schmutz JL, Barbaud A, et al. Cumulative incidence of, risk factors for, and outcome of dermatological complications of anti-TNF therapy in inflammatory bowel disease: a 14-year experience[J]. The American journal of gastroenterology. 2015,110(8):1186-96.

[22] Hiremath G, Duffy L, Leibowitz I. Infliximab-induced psoriasis in children with inflammatory bowel disease[J]. Journal of pediatric gastroenterology and nutrition. 2011,52(2):230-2.

[23] T M. <Skin reactions during anti-TNFa therapy for pediatric inflammatory bowel disease_a 2-year prospective study..pdf $>[\mathrm{J}]$. Inflammatory bowel diseases. 2014,20:1309-15.

[24] LA G, A G, RK C, G J, LJ G. Psoriasiform Skin Lesions Are Caused by Anti-TNF Agents Used for the Treatment of Inflammatory Bowel Disease[J]. Digestive diseases and sciences. 2015,60(11):3424-30.

[25] Afzali A, Wheat CL, Hu JK, Olerud JE, Lee SD. The association of psoriasiform rash with anti-tumor necrosis factor (anti-TNF) therapy in inflammatory bowel disease: a single academic center case series[J]. Journal of Crohn's \& colitis. 2014,8(6):480-8.

[26] Sondermann W, Herz S, Sody E, Korber A. Dermatological complications of therapy with biologics in inflammatory autoimmune diseases[J]. Journal der Deutschen Dermatologischen Gesellschaft = Journal of the German Society of Dermatology : JDDG. 2019,17(10):1029-37.

[27] Eickstaedt JB, Killpack L, Tung J, Davis D, Hand JL, Tollefson MM. Psoriasis and Psoriasiform Eruptions in Pediatric Patients with Inflammatory Bowel Disease Treated with Anti-Tumor Necrosis Factor Alpha Agents[J]. Pediatric dermatology. 2017,34(3):253-60.

[28] S KJ, AM T, M H, F S, D M. Anti-TNFa antibody-induced psoriasiform skin lesions in patients with inflammatory bowel disease: an Irish Cohort Study[J]. QJM : monthly journal of the Association of Physicians. 2017,110(6):379-82.

[29] Weizman AV, Sharma R, Afzal NM, Xu W, Walsh S, Stempak JM, et al. Stricturing and Fistulizing Crohn's Disease Is Associated with Anti-tumor Necrosis Factor-Induced Psoriasis in Patients with Inflammatory Bowel Disease[J]. Digestive diseases and sciences. 2018,63(9):2430-8.

[30] Peer FC, Miller A, Pavli P, Subramaniam K. Paradoxical psoriasiform reactions of anti-tumour necrosis factor therapy in inflammatory bowel disease patients[J]. Internal medicine journal. 2017,47(12):1445-8.

[31] Toussirot E, Aubin F. Paradoxical reactions under TNF-alpha blocking agents and other biological agents given for chronic immune-mediated diseases: an analytical and comprehensive overview[J]. RMD open. 2016,2(2):e000239.

[32] Pugliese D, Guidi L, Ferraro PM, Marzo M, Felice C, Celleno L, et al. Paradoxical psoriasis in a large cohort of patients with inflammatory bowel disease receiving treatment with anti-TNF alpha: 5-year follow-up study[J]. Alimentary pharmacology \& therapeutics. 2015,42(7):880-8.

[33] Denadai R, Teixeira FV, Steinwurz F, Romiti R, Saad-Hossne R. Induction or exacerbation of psoriatic lesions during anti-TNF-alpha therapy for inflammatory bowel disease: a systematic literature review based on 222 cases[J]. Journal of Crohn's \& colitis. 2013,7(7):517-24.

[34] Ko JM, Gottlieb AB, Kerbleski JF. Induction and exacerbation of psoriasis with TNF-blockade therapy: a review and analysis of 127 cases[J]. The Journal of dermatological treatment. 2009,20(2):100-8.

[35] Almutairi D, Sheasgreen C, Weizman A, Alavi A. Generalized Pustular Psoriasis Induced by Infliximab in a Patient With Inflammatory Bowel Disease[J]. Journal of cutaneous medicine and surgery. 2018,22(5):507-10.

[36] Ciccarelli F, De Martinis M, Sirufo MM, Ginaldi L. Psoriasis Induced by Anti-Tumor Necrosis Factor Alpha Agents: A Comprehensive Review of the Literature[J]. Acta dermatovenerologica Croatica : ADC. 2016,24(3):169-74.

[37] Li SJ, Perez-Chada LM, Merola JF. TNF Inhibitor-Induced Psoriasis: Proposed Algorithm for Treatment and Management[J]. Journal of psoriasis and psoriatic arthritis. 2019,4(2):70-80.

[38] Pariser DM, Bagel J, Gelfand JM, Korman NJ, Ritchlin CT, Strober BE, et al. National Psoriasis Foundation clinical consensus on disease severity[J]. Archives of dermatology. 2007,143(2):239-42.

[39] Wolf D, Skup M, Yang H, Fang AP, Kageleiry A, Chao J, et al. Clinical Outcomes Associated with Switching or Discontinuation from Anti-TNF Inhibitors for Nonmedical Reasons[J]. Clinical therapeutics. 2017,39(4):849-62 e6.

[40] Conrad C, Di Domizio J, Mylonas A, Belkhodja C, Demaria O, Navarini AA, et al. TNF blockade induces a dysregulated type I interferon response without autoimmunity in paradoxical psoriasis[J]. Nature communications. 2018,9(1):25.

[41] M P, A S, N Y, S V, F S. Development of psoriasis in IBD patients under TNF-antagonist therapy is associated neither with anti-TNF-antagonist antibodies nor trough levels[J]. Scandinavian journal of gastroenterology. 2016,51(12):1482-8.

[42] Vedak P, Kroshinsky D, St John J, Xavier RJ, Yajnik V, Ananthakrishnan AN. Genetic basis of TNF-alpha antagonist associated psoriasis in inflammatory bowel diseases: a genotype-phenotype analysis[J]. Alimentary pharmacology \& therapeutics. 2016,43(6):697-704. 
[43] Darrigade AS, Milpied B, Truchetet ME, Schaeverbeke T, Laharie D, Zerbib F, et al. Pattern and Severity of Psoriasiform Eruptions in Patients with Inflammatory Bowel Diseases, Arthritis or Skin Inflammatory Disorders Treated with TNF-alpha Inhibitors[J]. Acta dermato-venereologica. 2017,97(6):731-4.

[44] Harrison MJ, Dixon WG, Watson KD, King Y, Groves R, Hyrich KL, et al. Rates of new-onset psoriasis in patients with rheumatoid arthritis receiving anti-tumour necrosis factor alpha therapy: results from the British Society for Rheumatology Biologics Register[J]. Annals of the rheumatic diseases. 2009,68(2):209-15.

[45] Moran GW, Lim AW, Bailey JL, Dubeau MF, Leung Y, Devlin SM, et al. Review article: dermatological complications of immunosuppressive and antiTNF therapy in inflammatory bowel disease[J]. Alimentary pharmacology \& therapeutics. 2013,38(9):1002-24.

[46] Romeo AC, Ventimiglia M, Dipasquale V, Orlando A, Citrano M, Pellegrino S, et al. Effectiveness and safety of biologics in pediatric inflammatory boweldisease: Real-life data from the Sicilian Network[J]. Clinics and research in hepatology and gastroenterology. 2019.

[47] Tillack C, Ehmann LM, Friedrich M, Laubender RP, Papay P, Vogelsang H, et al. Anti-TNF antibody-induced psoriasiform skin lesions in patients with inflammatory bowel disease are characterised by interferon-gamma-expressing Th1 cells and IL-17A/IL-22-expressing Th17 cells and respond to anti-IL-12/IL23 antibody treatment[J]. Gut. 2014,63(4):567-77.

[48] Courbette O, Aupiais C, Viala J, Hugot JP, Louveau B, Chatenoud L, et al. Infliximab Paradoxical Psoriasis in a Cohort of Children With Inflammatory Bowel Disease[J]. Journal of pediatric gastroenterology and nutrition. 2019,69(2):189-93.

[49] Hellstrom AE, Farkkila M, Kolho KL. Infliximab-induced skin manifestations in patients with inflammatory bowel disease[J]. Scandinavian journal of gastroenterology. 2016,51(5):563-71.

[50] Soh JS, Yun WJ, Kim KJ, Won CH, Park SH, Yang DH, et al. Concomitant use of azathioprine/6-mercaptopurine decreases the risk of anti-TNFinduced skin lesions[J]. Inflammatory bowel diseases. 2015,21(4):832-9.

[51] Papamichael K, Vogelzang EH, Lambert J, Wolbink G, Cheifetz AS. Therapeutic drug monitoring with biologic agents in immune mediated inflammatory diseases[J]. Expert review of clinical immunology. 2019:1-12.

[52] Murias S, Magallares L, Albizuri F, Pascual-Salcedo D, Dreesen E, Mulleman D. Current Practices for Therapeutic Drug Monitoring of Biopharmaceuticals in Pediatrics[J]. Therapeutic drug monitoring. 2017,39(4):370-8.

[53] Gole B, Potocnik U. Pre-Treatment Biomarkers of Anti-Tumour Necrosis Factor Therapy Response in Crohn's Disease-A Systematic Review and Gene Ontology Analysis[J]. Cells. 2019,8(6).

[54] Melo FJ, Magina S. Clinical management of Anti-TNF-alpha-induced psoriasis or psoriasiform lesions in inflammatory bowel disease patients: a systematic review[J]. International journal of dermatology. 2018,57(12):1521-32.

[55] Protic M SA, Yawalkar N, et al. Development of psoriasis in IBD patients under TNF-antagonist therapy is associated neither with anti-TNF-antagonist antibodies nor trough levels[J]. Scandinavian journal of gastroenterology. 2016, 51:1482-8.

[56] Coutzac C CJ, Poullenot F, et al. Association between infliximab trough levels and the occurrence of paradoxical manifestations in patients with inflammatory bowel disease: a case-control study[J]. Journal of Crohn's \& colitis. 2015,9: 982-7.

[57] Vavricka SR, Gubler M, Gantenbein C, Spoerri M, Froehlich F, Seibold F, et al. Anti-TNF Treatment for Extraintestinal Manifestations of Inflammatory Bowel Disease in the Swiss IBD Cohort Study[J]. Inflammatory bowel diseases. 2017,23(7):1174-81.

\section{Tables}




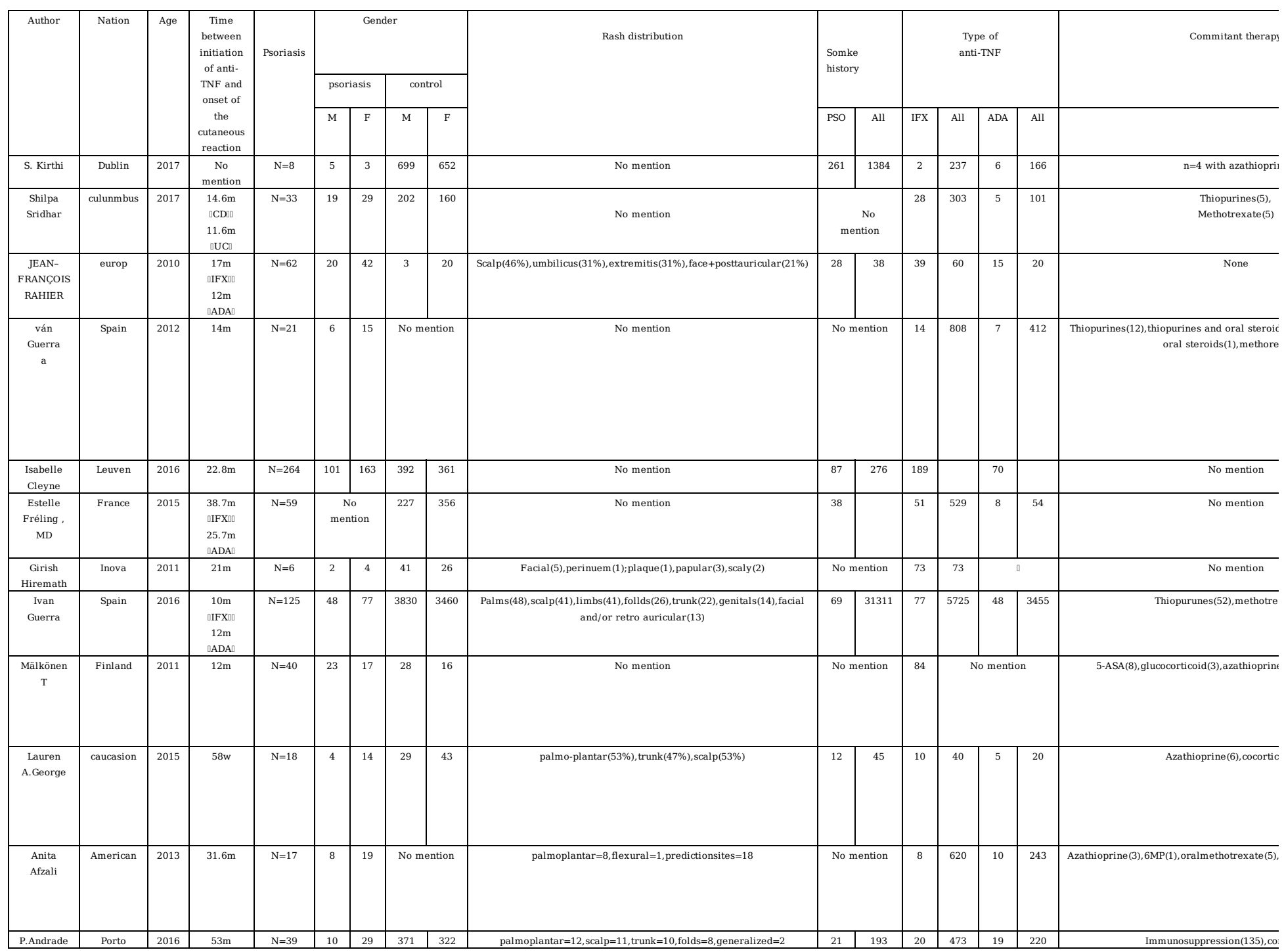

Table2: Summary of results.

\begin{tabular}{|c|c|c|c|c|c|c|c|}
\hline & Subgroup & No.of studies & $\begin{array}{l}\text { Summary OR(95\% } \\
\text { C)Fixed effect model }\end{array}$ & Q & P-heterogeneity & $\begin{array}{c}12 \\
\text { Statistic } \\
\%\end{array}$ & $\begin{array}{l}\text { Summary OR( }(95 \% \mathrm{Cl}) \\
\text { Random effect model }\end{array}$ \\
\hline Drug & & 8 & $0.665(0.52-0.85)$ & 10.71 & 0.129 & $37.70 \%$ & $0.651(0.452-0.936)$ \\
\hline \multirow[t]{3}{*}{ Gender } & & 9 & 1. $711(1.417-2.067)$ & 19. 33 & 0.013 & $58.60 \%$ & $1.957(1.346-2.846)$ \\
\hline & Pediatric & 3 & 1. $736(1.070-2.816)$ & 0.92 & 0.063 & $0.00 \%$ & $1.732(1.065-2.816)$ \\
\hline & Adults & 6 & $1.707(1.391-2.096)$ & 18.4 & 0.002 & $72.80 \%$ & 2. $122(1.261-3.571)$ \\
\hline \multirow[t]{3}{*}{ Smoke } & & 6 & $1.595(1.268-1.977)$ & 7. 22 & 0.205 & 30.8 & $1.679(1.237-2.279)$ \\
\hline & small & 3 & 2. $216(1.306-3.758)$ & 0.09 & 0.958 & $0.00 \%$ & 2. $222(1.312-3.762)$ \\
\hline & Large & 3 & $1.495(1.181-1.893)$ & 5.25 & 0.073 & $61.90 \%$ & $1.506(0.958-2.367)$ \\
\hline
\end{tabular}

\section{Figures}




\section{$(*:+$ represent +1 point, -1 represent -1 point, summary scores can be calculated $)$}

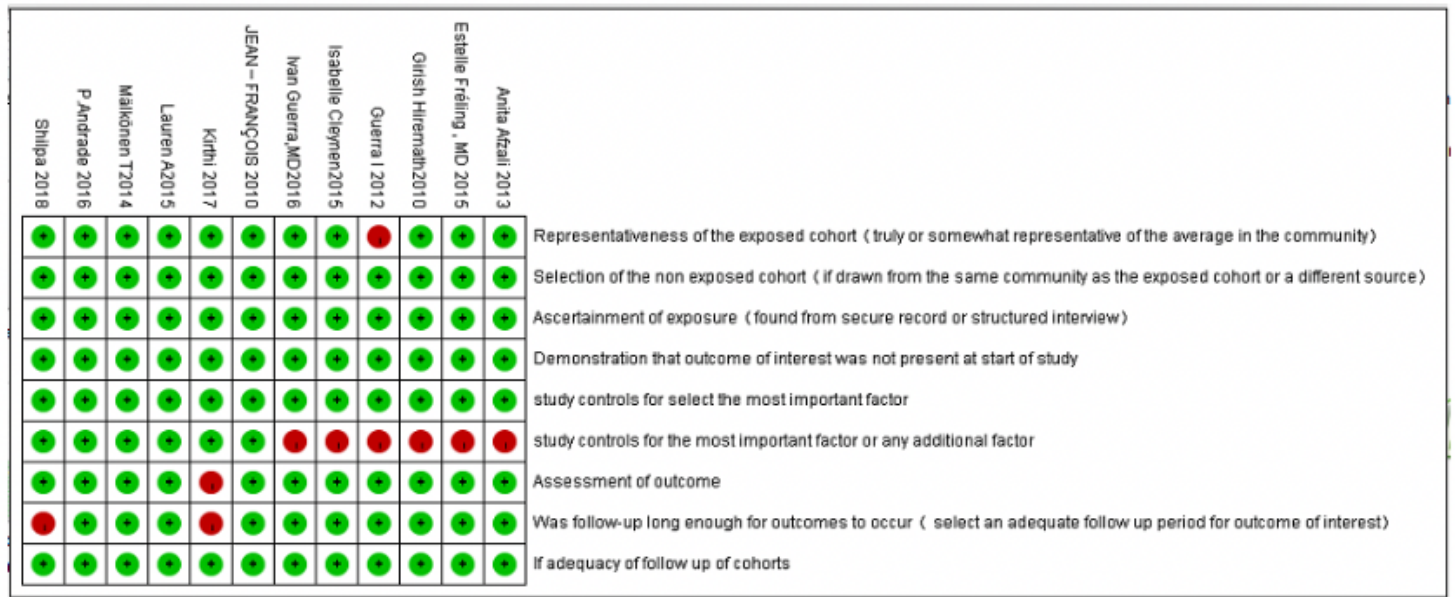

Figure 1

The NOS quaility elevation scores. $\rrbracket^{\star} \llbracket$ green circle represent +1 point, red circle represent -1 point, summary scores can be calculated $\rrbracket$

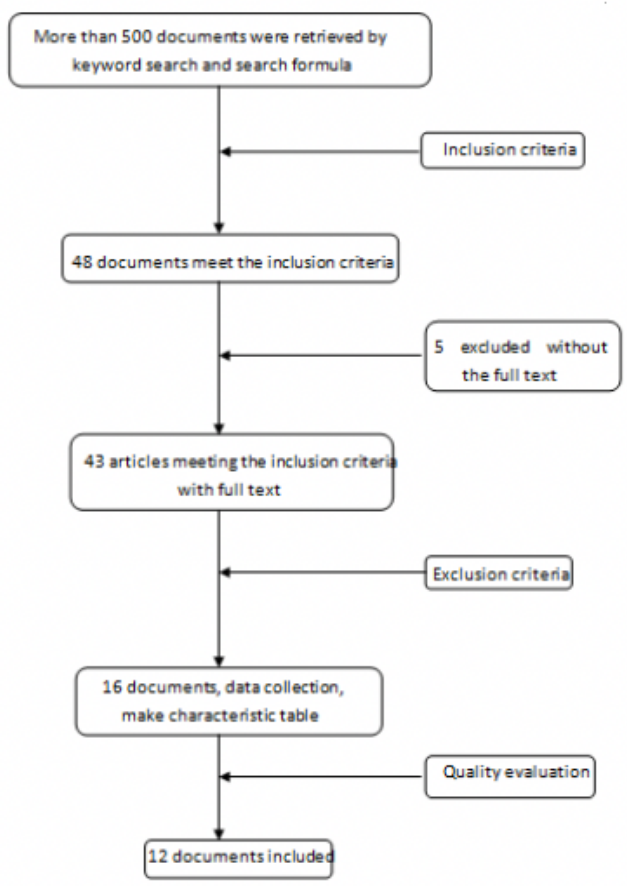

Figure 2

Flow diagram of literature screening 


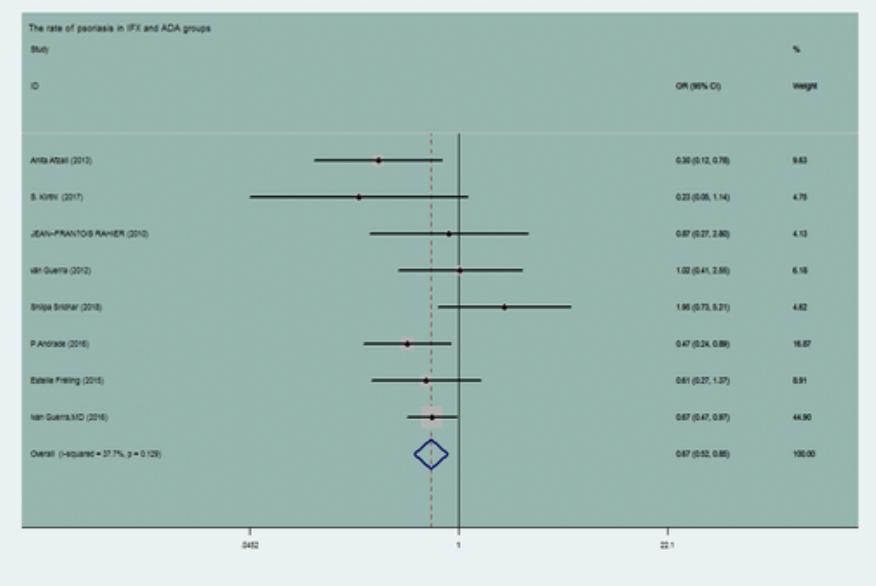

\section{Figure 3}

The association between IFX and ADA group

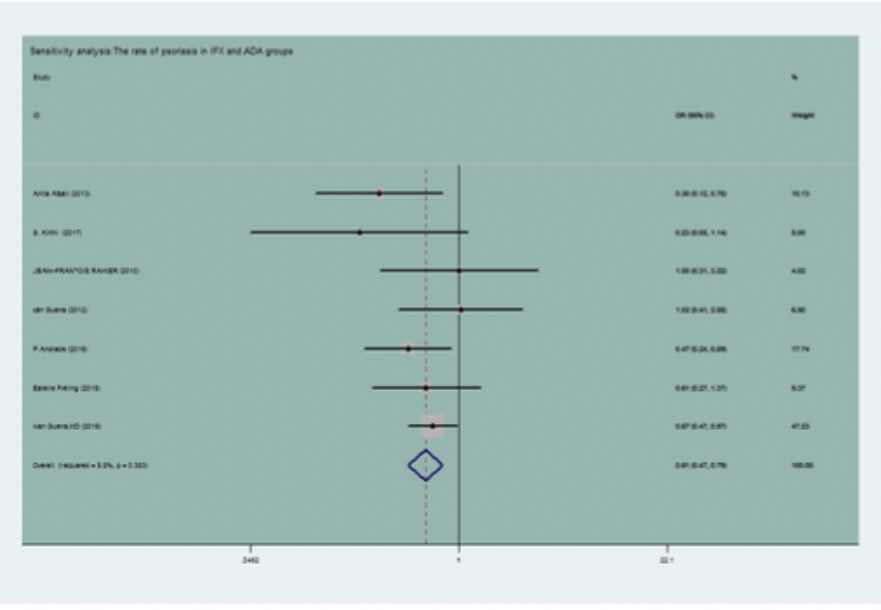

\section{Figure 4}

Sensitivity analysis: the association between the IFX and ADA group.

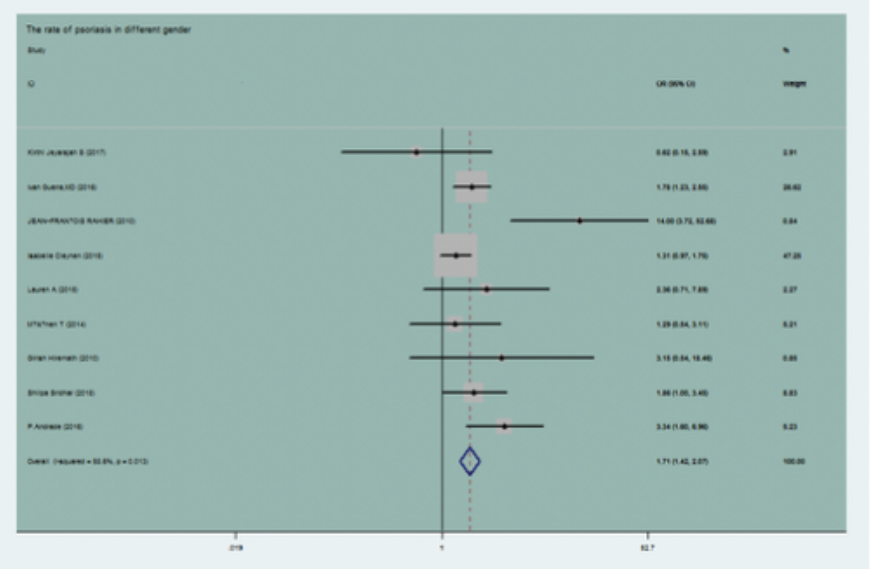

\section{Figure 5}

The association between different gender groups 


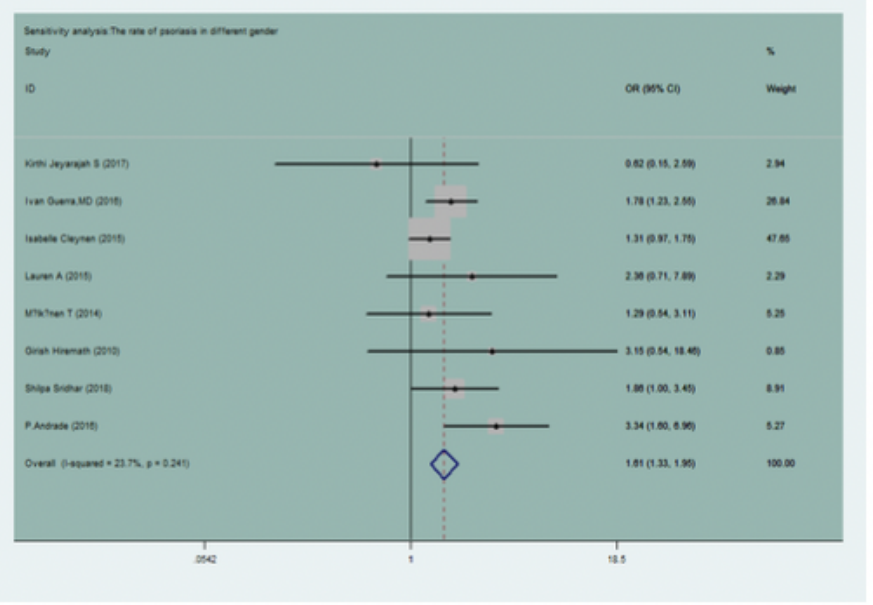

\section{Figure 6}

Sensitivity analysis: the association of psoriasis incidence between different gender groups

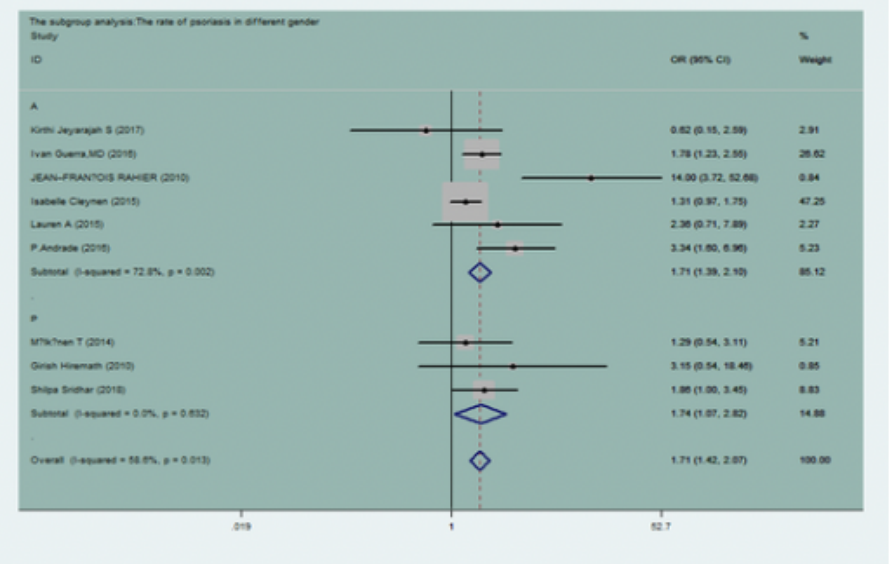

Figure 7

Subgroup analysis: the association of psoriasis between different gender groups

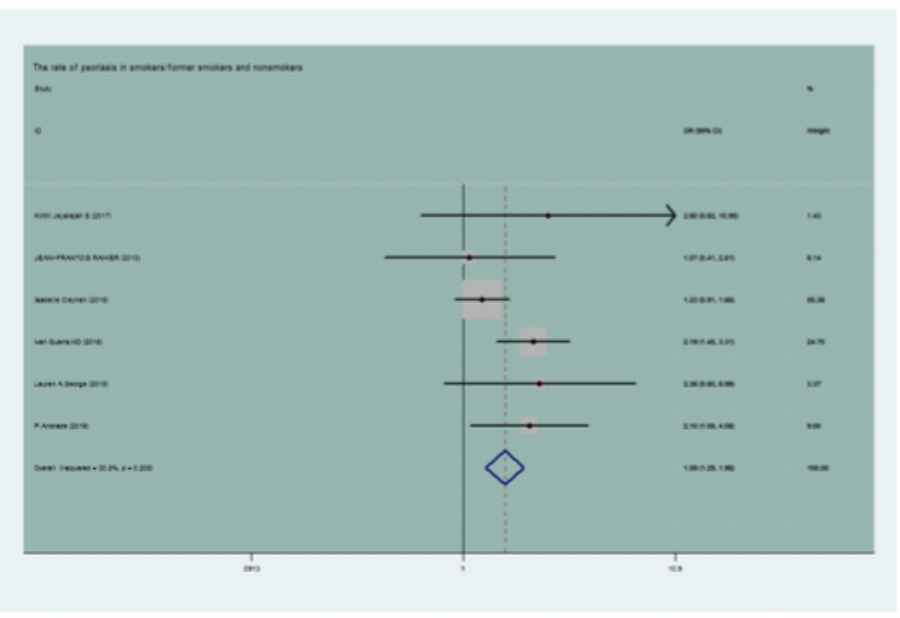

\section{Figure 8}

The association of incidence of psoriasis between smokers and nonsmokers 


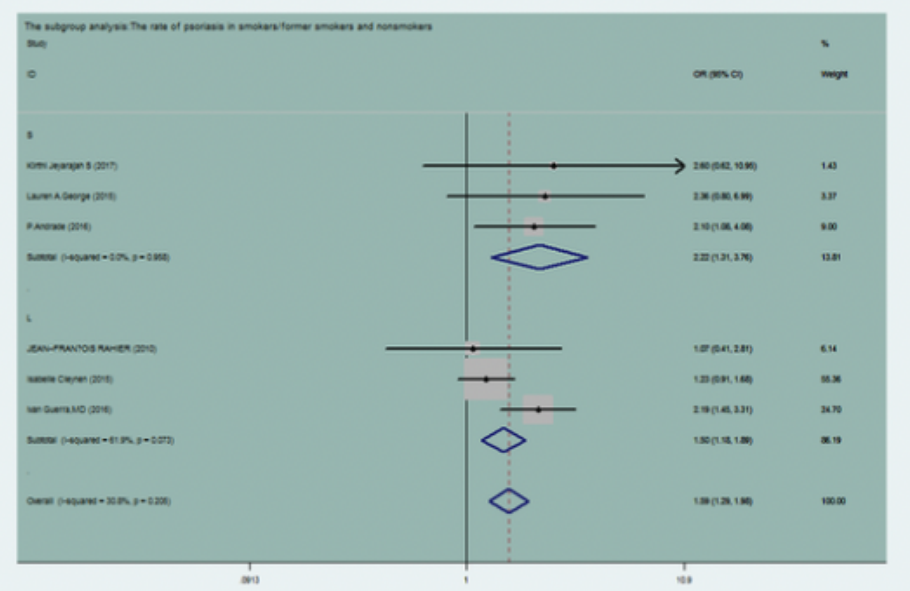

Figure 9

Subgroup analysis: the association of psoriasis between smokers and nonsmokers

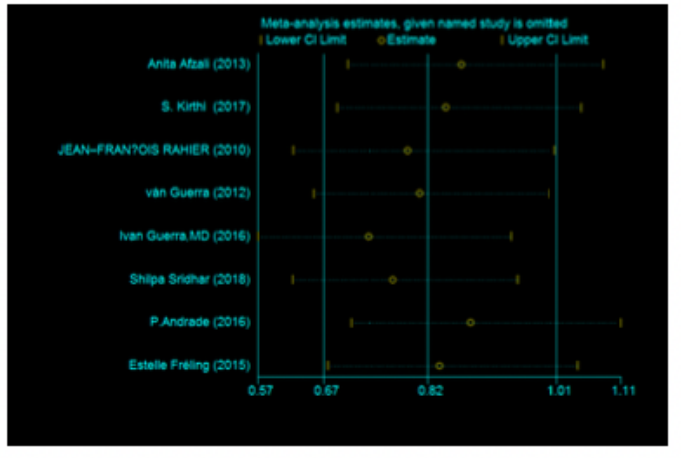

(a)

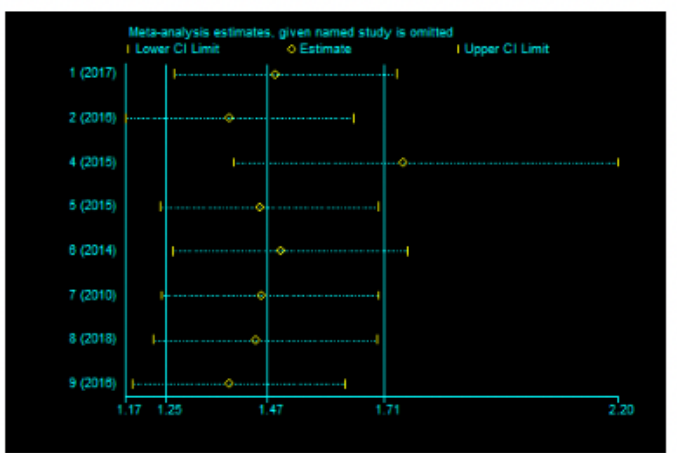

(c)

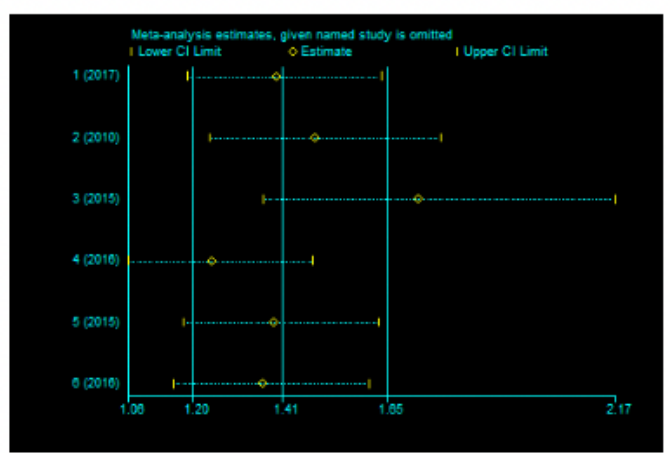

(b)

$(a, b, c)$ : Galbriarth chart to test the publication bias 


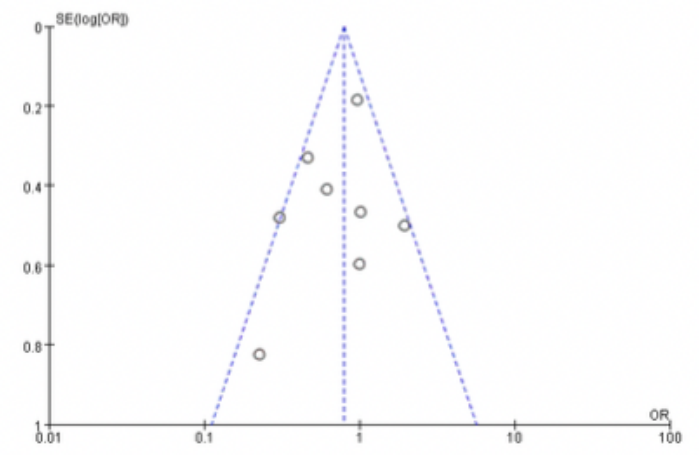

a

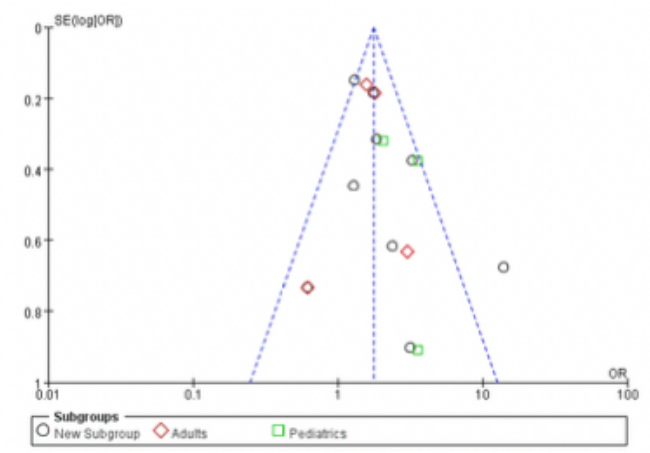

b

Figure 11

(a,b): Funnel polt

\section{Supplementary Files}

This is a list of supplementary files associated with this preprint. Click to download.

- ChecklistPRISMA2009.doc 